\title{
Assessment of provider-initiated HIV screening in Nigeria with sub-Saharan African comparison
}

Felix A. Ogbo ${ }^{1,7^{*}}$, Andrew Mogaji ${ }^{2}$, Pascal Ogeleka ${ }^{3}$, Kingsley E. Agho ${ }^{4}$, John Idoko ${ }^{5}$, Terver Zua Tule ${ }^{6}$ and Andrew Page ${ }^{1}$

\begin{abstract}
Background: Despite Nigeria's high HIV prevalence, voluntary testing and counselling rates remain low. UNAIDS/ WHO/CDC recommends provider-initiated testing and counselling (PITC) for HIV in settings with high HIV prevalence. We aimed to assess the acceptability and logistical feasibility of the PITC strategy among adolescents and adults in a secondary health care centre in Idekpa Benue state, Nigeria.
\end{abstract}

Method: All patients (aged $\geq 13$ years) who visited the out-patient department and antenatal care unit of General Hospital Idekpa, Benue state, Nigeria were offered PITC for HIV. The intervention was implemented by trained health professionals for the period spanning (June to December 2010).

Results: Among the 212 patients who were offered PITC for HIV, 199 (94\%) accepted HIV testing, 10 patients (4.7\%) opted out and 3 patients (1.4\%) were undecided. Of the 199 participants who were tested for HIV, 9\% were HIV seropositive. The PITC strategy was highly acceptable and feasible, and increased the number of patients who tested for HIV by 5\% compared to voluntary counselling and testing. Findings from this assessment were consistent with those from other sub-Saharan African countries (such as Uganda and South Africa).

Conclusion: PITC for HIV was highly acceptable and logistically feasible, and resulted in an increased rate of HIV testing among patients. Public health initiatives (such as the PITC strategy) that facilitate early detection of HIV and referral for early treatment should be encouraged for broader HIV control and prevention in Nigerian communities.

Keywords: Provider-initiated testing and counselling, HIV, AIDS, Benue State, Nigeria

\section{Background}

Globally, sub-Saharan Africa remains the region most affected by the human immunodeficiency virus/acquired immunodeficiency virus (HIV/AIDS) [1]. In 2015, subSaharan Africa countries (including Nigeria) accounted for approximately $75 \%$ of new HIV infections in adults [2]. Despite the introduction of voluntary counselling and testing (VCT) for HIV in sub-Saharan Africa countries, more than half $(60 \%)$ of people do not know their HIV status, and many living with HIV do not know that they are infected [3]. Consequently, majority of people

\footnotetext{
*Correspondence: felgbo@yahoo.co.uk

${ }^{1}$ Centre for Health Research, School of Medicine, Western Sydney University,

Campbelltown Campus, Locked Bag 1797, Penrith, NSW 2571, Australia

${ }^{7}$ General Hospital Idekpa, Ohimini Local Government Area, Benue State

Hospitals Management Board, Makurdi, Benue State, Nigeria

Full list of author information is available at the end of the article
}

living with HIV miss the opportunity for early detection and timely treatment, and also play an important role in the spread of the virus [4]. Of the annual incidence of HIV (approximately 2.6 million per year) worldwide [2], an estimated $40-90 \%$ of these cases experience symptoms of acute HIV infection, and a substantial number would seek medical care [5-7]. However, symptoms of acute HIV infection are often missed by primary care clinicians because these symptoms are similar to those of influenza and other viral illnesses [8].

To increase the number of people who test for HIV, the Joint United Nations Programme on HIV/AIDS/World Health Organization (UNAIDS/WHO, including the Centre for Disease Control, CDC) recommends provider-initiated testing and counselling (PITC) strategy for all patients aged $\geq 13$ years. PITC refers to a routine offer of HIV testing 
and counselling for all patients who visit a health facility by a health professional (particularly in HIV endemic communities), regardless of the presenting symptoms, and without a separate written consent [9]. This intervention presents an important opportunity for preventive health care services in patients - a major tenet of primary care $[10,11]$. The PITC strategy has been employed successfully in developing countries with high HIV prevalence. For example, studies from Uganda [12], Zambia [13] and South Africa [14] found that PITC strategy (opt-out) increased the proportion of patients who tested for HIV and the number of patients who commenced anti-retroviral therapy. These studies also showed that PITC was highly acceptable and feasible in the health facility. Similarly, a study from Nigeria conducted among a cohort of university students found that the optout strategy was feasible and highly acceptable [15].

In 2007, the Federal Ministry of Health in Nigeria adopted the PITC strategy; however, evidence suggests that many health care providers in Benue State were unaware of the PITC strategy. Benue State of Nigeria (at the time of this assessment) was one of the states with the highest HIV prevalence $(5.0-10.6 \%)$ in the country, with variability in transmission rates in different Local Government Areas of the state [16]. Although the PITC strategy was implemented for pregnant women during antenatal care visits, this initiative was not broadened to include other adults who presented to the hospital as recommended.

Following the UNAIDS/WHO/CDC recommendation of PITC for HIV $[9,10]$, our health care center (General Hospital Idekpa) in Benue State, Nigeria recognised the need to increase HIV screening among adolescents and adults, and was the first in the state to incorporate routine provider-initiated HIV screening in all consultations for patients, including pregnant women. In this study, we aimed to assess the acceptability and logistical feasibility of the PITC strategy (under routine operational environment) among adolescents and adults who presented to the general out-patient clinic and antenatal care of General Hospital Idekpa in Benue State, Nigeria.

The implementation of the PITC approach in our local health facility was based on findings from previously published studies from Uganda [12], Botswana [17] and South Africa [18]. The PITC strategy was also implemented to provide context-specific evidence to determine the extent to which patients accepted HIV testing, and for public health practitioners to advocate for effective initiatives to improve the uptake of HIV testing in Benue State, Nigeria. Additionally, Nigeria is the largest recipient of developmental assistance for Health, including assistance for HIV prevention and control programmes [19]. Findings from this study will not only be of interest to public health agencies in Nigeria, but will also be of interest internationally to define the scope to which the PITC approach is context-specific.

\section{Methods}

\section{Study setting}

The study was implemented in a public health facility in Idekpa, Ohimini Local Government Area, Benue State of Nigeria. The Local Government Area has one comprehensive health care centre and seven primary health care centres with a population of 70,688 people based on the 2006 national census. In January 2010, the Benue State Government collaborated with the World Bank and Africa Development Bank to upgrade the comprehensive health centre in Idekpa to a secondary health care facility (General Hospital Idekpa), which is currently funded and managed by the Benue State Government. Before the upgrade, VCT was the standard approach to HIV detection, provided by a trained nurse and other lay health workers.

Approximately 900 patients visited the comprehensive health centre annually; of which, an estimated 70 patients (including pregnant women) were tested for HIV (8\%), using the VCT approach. Following the upgrade of the comprehensive health centre to a General Hospital, an estimated 470 patients presented to the out-patient department and antenatal care unit within 6 months (June-December, 2010). Of these 212 patients (adolescents or adults aged $\geq 13$ years) were eligible for the PITC strategy during this time interval, the period for this assessment. Patients (adults and children) who were HIV positive and taking anti-retroviral medications were not eligible. These patients were identified because they notified the attending clinical staff of their HIV positive status, and were counselled on the importance of drug compliance and follow-up care. In the six months following the implementation of the PITC strategy, an estimated 1,030 patients presented to the General Hospital. The increase in the latter was due to various community mobilisation activities implemented by the Benue State Government to create awareness among community members about the General Hospital upgrade.

In General Hospital Idekpa, health care services are provided at a cost (out-of-pocket), and are delivered by doctors, pharmacists, and trained nurses and midwives, including trained laboratory technicians. These service providers were trained in HIV/AIDS counselling and testing modalities. The PITC strategy for HIV screening was introduced in the health facility as a standard approach to health care, and counselling was provided by doctors trained nurses and midwives, consistent with the WHO/UNAIDS/CDC recommendation. At the start of the intervention in June 2010, the average patient's turnout was 5-10 per week with adequate documentation of HIV testing and counselling.

\section{Implementation of the PITC strategy}

The PITC strategy was implemented for all visiting eligible patients (adolescents or adults aged $\geq 13$ years; 
$N=212)$ in the out-patient department and antenatal unit of General Hospital Idekpa, Benue State of Nigeria for the period (June-December, 2010). The implementation of this strategy aimed to assess the acceptability of HIV testing and counselling under normal operational and management conditions in the health facility to provide local data to relevant oragnisations. Random selection was not feasible because the strategy was aimed at all presenting patients. The implementation of the PITC strategy was facilitated by the health management team and first-line health professionals at the hospital. This approach made it possible to assess the logistical feasibility of the PITC strategy under routine operational environment of a secondary health care facility in a developing country.

The main outcome was the acceptability and logistical feasibility of routine provider-initiated HIV screening of new patients. The acceptability of the HIV test was measured as the proportion of patients who were offered HIV counselling and testing relative to those eligible for the test. Logistical feasibility was assessed as the proportion of patients who accepted HCT relative to those who were offered the test [12]. We also described the sample in terms of the patient's gender, age, marital status and the proportion of those who have not being previously tested for HIV, including the test results, similar to previously published articles $[12,14]$.

\section{The provider initiated testing and counselling (PITC) strategy for HIV}

The PITC strategy employed in this assessment was a modified version of the 'ACTS' approach, which includes four brief steps: assess, obtain consent, test, and provide supportive services [20]. In this study, a doctor or a trained nurse/midwife (trained in HIV testing and counselling) conducted the assessment to identify eligible participants, obtained verbal consent, and offered HIV testing as a standard part of medical care for all eligible patients, regardless of the patient's presenting symptoms. The patients were allowed to decline or opt-out of the current testing. According to the WHO guideline for the provider-initiated HIV screening, verbal consent is allowed for the test to be performed [11]. Abbreviated pre-test counselling was also conducted, which consisted of informing patients about the HIV disease and that a person can only detect the disease by doing an HIV test; a recommendation that they should test for HIV at the index consultation; and patients would receive relevant services whether or not the test is positive or negative. If the person agreed, the doctor or the nurse/midwife would do a brief test readiness assessment, obtain verbal informed consent, and send the patient for a rapid HIV test along with other investigations that the patient may require. A patient refusal to take the test did not affect the quality of health service received, nor did it affect the patient-health provider relationship or confidentiality. Where a test offer was refused, further opportunities were created for the patient to either access a VCT or PITC in the future.

Rapid HIV testing was used, and the sequential (serial) rapid testing algorithm was employed where Determine HIV-1/2 assay kit (Abbott Laboratories, Illinois, United States of America) was used for screening; Uni-Gold test kit (Trinity Biotech, Wicklow, Ireland) was used as a confirmatory test, and HIV-1/2 STAT-PAK Dipstick assay (Chembio Diagnostic Systems Inc., New York, USA) was employed as the tie-breaker.

An HIV-negative result with the Determine assay was reported as negative. An HIV-positive result with the Determine assay was confirmed with the Uni-Gold test, and was reported as positive if both (Determine and Uni-Gold) tests gave positive results. If the Determine and Uni-Gold test results were discordant, the sample was subjected to a third test - the STAT-PAK assay. The result was reported as positive if the STAT-PAK assay result was positive, and negative if both Uni-Gold test and STAT-PAK assay results were negative.

Patients received post-test counseling and test results on the same day - within $60 \mathrm{~min}$ in most cases. Test results were provided to patients by the medical team (doctor or nurse/midwife). Health care providers encouraged patients to disclose their HIV status to their sexual partners and/or family members when they felt ready to do so. Additionally, health care providers assisted with disclosures if requested by the patient. The disclosures of HIV test results to either sexual partners or family members/friends were conducted by health professionals who had undergone appropriate training in HIV counselling. All patients, (whether HIV-negative or HIV-positive) were counselled on risk reduction after the test. Patients who tested HIV-positive were also provided information on available HIV/AIDS care and support, and co-trimoxazole (septrin) prophylaxis, and tuberculosis screening was initiated on diagnosis, consistent with the National HIV treatment guidelines. On discharge (if admitted) or after counselling, patients who tested HIV-positive were given referrals to HIV/AIDS specialised clinics (based on patient's choice) for followup care.

\section{Data collection and analysis}

Data collected from participants who were offered PITC included: age, gender, educational level, marital status, religion, history of HIV testing, most recent sexual partners and knowledge of HIV status of sexual partner (for those who reported sexual contact within the last six months prior to presentation at the hospital). Reasons for declining the current testing, and result of the HIV 
test (for those who accepted the test) were also documented. Frequencies and corresponding percentages with confidence intervals were calculated using Fisher's exact. All analyses were conducted in SPSS 2007 version 15.0.

\section{Ethics}

Ethical approval for this assessment and subsequent publication of findings was obtained from the Benue State Ministry of Health Human Research Ethics Committee. All patients and caregivers (on behalf of children 13-17 years) were aware that as part of the clinical care, their demographic and clinical data were registered in paper-based clinic registers, stored in the hospital medical registry. Data employed for this assessment were anonymous, and the ethics committee did not require that the investigators sought written informed consent from patients based on the WHO PITC guideline. All patients or caregivers provided verbal informed consent to health care workers before they were allowed to participate.

\section{Results}

The introduction of the PITC strategy (from June to December 2010) increased the number of patients who tested for HIV in the health facility compared to the VCT approach (i.e., from $8.0 \%$ in the 12 months prior to the upgrade of the health centre to $13.3 \%$ in the period of this assessment). The $8.0 \%$ was estimated based on the total number of patients seen in the comprehensive health centre in 12 months (900 patients) and the number of patients tested for HIV (70 patients) over the same period, using the VCT approach. The $13.3 \%$ was estimated based on the total number of patients seen at the General Hospital (1,500 patients) annually and 212 patients tested for HIV over the study period, using the PITC strategy. This proportion (13.3\%) underestimates the actual impact of the PITC strategy because it was implemented for six months compared to the VCT which was conducted for one year. Of the 212 patients who were offered PITC for HIV, 199 (93.9\%) accepted the HIV test, 10 (4.7\%) opted out and $3(1.4 \%)$ were undecided (Table 1). The reasons for opting out of the test included: being sure of their HIV status $(n=6)$; to inform husband/partner before taking the HIV test $(n=3)$ and not being ready for HIV testing $(n=1)$.

More than half of the patients who accepted the HIV test reported a steady sexual partner in the six months preceding their presentation to the hospital (54\%). Almost a quarter of patients reported being previously tested for HIV, and knew their HIV status (24\%). Among the patients who tested for HIV, many tested negative (91\%), while nine percent tested HIV positive (Table 2).
Table 1 Characteristics of the study population

\begin{tabular}{|c|c|c|}
\hline Characteristics & $N^{a}=212(\%)$ & $N^{b}=199(\%)$ \\
\hline \multicolumn{3}{|l|}{ Acceptability of HIV test } \\
\hline Yes & $199(93.9)$ & \\
\hline No & $10(4.7)$ & \\
\hline Undecided & $3(1.4)$ & \\
\hline \multicolumn{3}{|l|}{ Gender } \\
\hline Female & & $131(65.9)$ \\
\hline Male & & $68(34.1)$ \\
\hline \multicolumn{3}{|l|}{ Age (years) } \\
\hline $13-20$ & & $29(14.5)$ \\
\hline $21-30$ & & $52(26.1)$ \\
\hline $31-40$ & & $43(21.6)$ \\
\hline $41-50$ & & $35(17.6)$ \\
\hline $51-60$ & & $20(10.1)$ \\
\hline $60+$ & & $20(10.1)$ \\
\hline \multicolumn{3}{|l|}{ Marital status } \\
\hline Single & & $119(59.8)$ \\
\hline Married & & $48(24.1)$ \\
\hline Separated/divorced & & $8(4.0)$ \\
\hline Widowed & & $24(12.1)$ \\
\hline \multicolumn{3}{|l|}{ Education } \\
\hline No education & & $47(23.6)$ \\
\hline Primary education & & $67(33.7)$ \\
\hline Secondary education & & $65(32.7)$ \\
\hline Tertiary education & & $20(10.0)$ \\
\hline \multicolumn{3}{|l|}{ Religion } \\
\hline Christian & & $192(96.5)$ \\
\hline Muslim & & $7(3.5)$ \\
\hline \multicolumn{3}{|l|}{ Previously tested for HIV } \\
\hline Yes & & $57(28.6)$ \\
\hline No & & $142(71.4)$ \\
\hline \multicolumn{3}{|c|}{ Result of previous HIV test, if known } \\
\hline Negative & & $47(23.6)$ \\
\hline Unknown & & $10(5.0)$ \\
\hline \multicolumn{3}{|c|}{ Most recent (last 6 months) sexual partner } \\
\hline Husband & & $30(15.1)$ \\
\hline Steady partner & & $107(53.8)$ \\
\hline Casual partner & & $4(2.0)$ \\
\hline None & & $58(29.1)$ \\
\hline \multicolumn{3}{|l|}{ Result of HIV test } \\
\hline Positive & & $18(9.0)$ \\
\hline Negative & & $181(91.0)$ \\
\hline
\end{tabular}

$\mathrm{N}^{\mathrm{a}}$ : Total number of participants who were offered HIV testing

$\mathrm{N}^{\mathrm{b}}$ : Total number of participants who accepted HIV testing 
Table 2 Prevalence of HIV cases in the population by study factor

\begin{tabular}{|c|c|c|c|}
\hline Characteristics & HIV cases & (\%) 95\%(LCI-UCl) & $P$ value \\
\hline \multicolumn{4}{|l|}{ Gender } \\
\hline Female & 14 & $10.6(5.9-17.2)$ & 0.434 \\
\hline Male & 4 & $5.8(1.6-14.4)$ & \\
\hline \multicolumn{4}{|l|}{ Age (years) } \\
\hline $13-20$ & 1 & $0.3(0.1-17.7)$ & 0.430 \\
\hline $21-30$ & 5 & $9.6(3.1-21.0)$ & \\
\hline $31-40$ & 5 & $11.6(3.9-25.1)$ & \\
\hline $41-50$ & 1 & $2.9(0.1-14.9)$ & \\
\hline $51-60$ & 4 & $20.0(5.7-43.7)$ & \\
\hline $60+$ & 2 & $10.0(1.2-31.7)$ & \\
\hline \multicolumn{4}{|l|}{ Marital status } \\
\hline Single & 9 & $7.5(3.5-13.9)$ & 0.048 \\
\hline Married & 2 & $4.6(0.1-14.2)$ & \\
\hline Separated/divorced & 3 & $37.5(8.5-75.5)$ & \\
\hline Widowed & 4 & $17.7(4.7-37.3)$ & \\
\hline \multicolumn{4}{|l|}{ Education } \\
\hline No education & 4 & $8.5(2.7-20.4)$ & 0.255 \\
\hline Primary education & 10 & $14.9(7.4-25.7)$ & \\
\hline Secondary education & 4 & $6.1(1.7-15.0)$ & \\
\hline Tertiary education & 0 & - & \\
\hline \multicolumn{4}{|l|}{ Religion } \\
\hline Christian & 18 & $9.3(5.7-14.4)$ & 0.541 \\
\hline Muslim & 0 & - & \\
\hline
\end{tabular}

LCI Lower Confidence Interval, UCI Upper Confidence Interval

$\mathrm{N}$ : Total number of participants who accepted HIV testing

$\%$ : Proportions of HIV cases with 95\% Confidence Intervals (Cls)

The PITC strategy was logistically feasible in General Hospital Idekpa, Benue Sate of Nigeria because HIV testing was affordable and quick, and counselling was provided by health care professionals (doctors and nurses/midwives). These findings were compared to evidence from selected sub-Saharan African countries (Table 3).
Discussion of the study findings, with sub-Saharan African comparison

A well-coordinated routine PITC strategy that was integrated into the general health care services, and delivered by health care professionals in a secondary health facility in Benue State Nigeria was highly acceptable and logistically feasible, with majority of patients opting for HIV testing who previously did not know their HIV status. In comparing the 6-months period of the PITC implementation in our facility with the epoch (one year) pre-implementation using the VCT approach, the PITC strategy appears to be more effective in the hospital environment. Consistent with previous studies from Benue state Nigeria [21, 22], this study found a higher prevalence of HIV testing among participants who had primary/secondary education compared to those who had no schooling. In addition, the PITC strategy provided information on HIV transmission and prevention (during the pre- and post-test counseling) to the patients and the community. Our findings were consistent with those from other developing countries (such as South Africa, Uganda, Rwanda, Zambia and Botswana) with high prevalence of HIV. These studies also found that PITC was highly acceptable and feasible, and increased the proportion of patients who tested for HIV compared to the VCT approach [12, 17, 18, 23, 24].

The high acceptance of the PITC strategy for HIV testing, with an option to decline has been shown to reduce maternal-to-child transmission of HIV in populations $[17,25]$. Consistent with a study from South Africa [26], our study found that the reasons as to why patients declined to take the HIV test included: being tested previously; the believe that they were not at risk; the need to obtain consent from their partner/husband before taking the HIV test; and not being ready for the test. Additional reasons for why patients may accept or refuse an HIV test using the PITC strategy include cost of the HIV test [12] or a lack of money [27]; level of referral system, access to HIV specialised care and skill of the health care provider [14]; human resources at the health facility [12]; and confidentiality [28].

Table 3 Summary of provided initiated HIV testing and counselling implementation results from selected sub-Saharan African countries

\begin{tabular}{llllllllll}
\hline Authors & Country & Study setting & $\mathrm{N}^{a}$ & $\mathrm{~N}^{\mathrm{b}}$ & $\%(\mathrm{LCl}-\mathrm{UCl})$ & $\mathrm{N}^{c}$ & $\%(\mathrm{LCl}-\mathrm{UCl})$ & $\mathrm{N}^{d}$ & $\%(\mathrm{LCl}-\mathrm{UCl})$ \\
\hline Kharsany et al., 2010 [26] & South Africa & Hospital-based & 5612 & 2439 & $43.0(42.0-45.0)$ & 3173 & $57.0(55.0-58.0)$ & 1378 & $56.0(55.0-58.0)$ \\
Wanyenze et al., 2008 [12] & Uganda & Hospital-based & 51642 & 50649 & $98.0(98.0-98.0)$ & 993 & $2.0(2.0-2.0)$ & 12107 & $0.24(24.0-24.0)$ \\
Leon et al., 2010 [14] & South Africa & Hospital-based & 2326 & 1752 & $75.0(74.0-77.0)$ & 574 & $25.0(23.0-26.0)$ & 326 & $0.19(17.0-21.0)$ \\
Bassett et al., 2007 [18] & South Africa & Hospital-based & 2912 & 1414 & $49.0(47.0-50.0)$ & 1498 & $51.0(50.0-53.0)$ & 463 & $0.33(30.0-35.0)$ \\
ljadunola et al., 2011 [15] & Nigeria & University & 252 & 251 & $99.6(98.0-1.00)$ & 1 & $40.0(0.0-2.0)$ & 0 & $0.00(0.0-1.0)$ \\
Topp et at., 2011 [24] & Zambia & Hospital-based & 41861 & 31197 & $75.0(74.0-75.0)$ & 10644 & $34.0(34.0-35.0)$ & 6572 & $0.21(21.0-22.0)$ \\
\hline
\end{tabular}

$\mathrm{LCl}$ Lower Confidence Interval, UCI Upper Confidence Interval

$\mathrm{N}^{\mathrm{a}}$ : Number of test offered; $\mathrm{N}^{\mathrm{b}}$ : Number of test accepted; $\mathrm{N}^{\mathrm{c}}$ : Number of offered test declined; $\mathrm{N}^{\mathrm{d}}$ : Number of HIV positive 
Although patients paid for the HIV test at the facility (at a cost of one hundred and fifty naira, equivalent of one United Sates dollar - based on the 2010 exchange rate), the study found that a large proportion of patients accepted the HIV test compared to a study from South Africa [18]. The South African study reported that more than half of the patients declined to be tested (51\%). This refusal may be due to the fee charged for the HIV test or a reflection of the patient's right to decline the test - part of the ethical implication of the PITC strategy. The payment for the HIV testing (rapid test kits) was employed by the hospital management team because the implementation of a fully funded HIV program was still under consideration by relevant health and donor agencies at the time of the assessment. Despite this payment, a high acceptance rate was observed in this study, consistent with findings from Uganda which introduced the free PITC strategy [12]. The high acceptance rate in our facility was likely to be multifactorial. Firstly, patients were probably less apprehensive in participating in the PITC approach because the strategy may have been perceived by patients as a "standard of care" offered to all patients, thereby reducing the risk of stigma and other adverse social concerns attached to HIV. In many communities, stigma and discrimination continue to be the prevailing response to HIV/AIDS diagnosis, particularly in the VCT (opt-in) approach, probably because special HIV clinics are used for the VCT strategy [29]. Secondly, community sensitization and availability of the on-site rapid HIV testing may have played a significant role in the high acceptance rate and HIV testing among patients. In many sub-Saharan African countries (including Nigeria), health care professionals are well respected by the community and are seen as change agents. This perception may have played a role in this study as health professionals directly offered the HIV test to patients, similar to other studies from sub-Saharan Africa [12, 18, 23].

Findings from this study have operational connotations beyond the HIV testing and counselling strategy as it relates to the management of staffing resources. In this study, doctors and nurses integrated HIV screening into their medical consultations, taking on a duty previously performed by a less skilled worker or trained HIV counsellor. The strategy employed in this study is different from 'task shifting' recommended by the WHO, where responsibilities are referred down to junior health workers in order to reduce consultation times for treatment, care and support of HIV patients [30, 31]. The PITC approach capitalised on existing staffing resources, particularly in resource poor settings such as Nigeria because it was incorporated into routine medical consultations performed by skilled health professionals, consistent with previous studies $[12,14]$. The expansion in doctors and nurses' roles was achieved with meetings of the hospital management team and front-line clinical staff. This strategy extended consultation times by approximately ten to fifteen minutes on average, and was similar to a study from Rwanda [23]. However, in communities where trained health personnel (such as doctors and nurses) are limited, trained lay health workers who regularly consult patients can initiate the PITC strategy to increase HIV testing and coverage [32].

Policies and procedures to promote early detection of HIV infection in adolescents and adults using the routine PITC strategy have raised ethical concerns with regard to the definition and implementation of the strategy, particularly in settings with high proportion of illiteracy, poverty, gender disparities, poor access to treatment and fragile healthcare structure [33, 34]. Despite these concerns, the PITC is voluntary, and involves the "three Cs" - informed consent, counselling and confidentiality - that must be observed. In the optout approach, adolescents and adults must explicitly decline the HIV test after receiving the pre-test education. In contrast, the patient must consent to the HIV test after receiving the pre-test education in the opt-in strategy [35]. In comparing the VCT approach to PITC, a population-based survey from Botswana found that PITC reduced barriers to HIV testing, increased the proportion of people who tested for HIV and was broadly supported by the community [17]. Efforts to implement the PITC strategy must strictly consider and adhere to relevant guidelines with regard to protecting individual confidentiality, informed consent and ensuring adequate linkages to treatment, care and support [36].

Despite the introduction of the VCT and PITC approaches in the hospital environment to increase uptake of HIV testing, studies from developing contexts have shown that community-based HIV testing and counselling approaches (that is, testing outside of health facilities) had a higher coverage and uptake, and identified people living with HIV who were unware of their HIV status compared to hospital-based HIV testing strategies [37-39]. These findings highlight the need for a comprehensive strategy that involves facility- and communitybased HIV testing approaches to effectively increase uptake of HIV counselling and testing in Nigeria's communities. Aspects of these initiatives can include a complete roll-out of the PITC strategy, mobile testing, door-todoor testing, self-testing, index testing, workplace testing, school-based testing, church-based testing and outreach testing, streamlined with local resources and galvanised with political support. Previous reports have revealed that these measures were effective in increasing the uptake of HIV testing [39, 40].

A number of challenges were encountered during the implementation of the opt-out strategy. Firstly, the 
clinics were understaffed with regard to nurses. In many settings, nurses are usually trained in HIV counselling to improve HIV testing rate; but while nurses in our facility were trained in HIV counselling, the increase in clinic workload due to staff shortages resulted in reluctance among nurses to take up additional duty of the PITC strategy. Studies on the experiences of health care workers in relation to the implementation of PITC strategy are warranted. Secondly, the VCT approach should have been assessed at the General Hospital because this information would have been very useful for effective comparison of both VCT and PITC approaches. Thirdly, studies from South Africa and Burkina Faso found lower prevalence of HIV testing among males [18, 41]; however, our assessment of the PITC strategy among males was challenging because fewer males compared to females presented to the facility. This male pattern of health seeking behaviour has been reported elsewhere [42-44]. Fourthly, the HIV prevalence observed in this study may not be representative of the overall HIV prevalence in the community because primary health centres in the local environment also provided HIV VCT services to patients. Despite these limitations, our study provides local evidence on the acceptability and logistical feasibility of the PITC strategy in Nigeria for informing HIV prevention and control initiatives. Additionally, all patients (severally ill or not) who presented to the out-patient department were included in this assessment, and this approach ensures early detection of HIV seropositive patients and subsequent referral for early treatment and care, when compared to a previously published study [18].

\section{Conclusion}

The PITC strategy that was incorporated into the outpatient clinic and antenatal care unit of a secondary health care centre in Idekpa, Nigeria was highly acceptable and logistically feasible. The strategy also increased HIV testing rate among people who previously have not been tested for HIV (by 5\%) compared to the VCT approach. Interventions and policy responses that facilitate health care centres to initiate the PITC strategy in Nigeria should be encouraged, but this approach must consider individual confidentiality, informed consent and counselling as well as the local context.

\section{Abbreviations \\ AIDS: Acquired immunodeficiency virus; CDC: Centre for disease control; HCT: HIV counselling and testing; HIV: Human immunodeficiency virus; PITC: Provided initiated testing and counselling; UNAIDS: Joint United Nations Programme on HIV/AIDS; VCT: Voluntary counselling and testing; WHO: World Health Oragnisation}

\section{Acknowledgements}

The authors are grateful to Late Mrs Joyce Adah, Andrew Odeh, Alexander Egboche, Christiana Ameh, Apeh Michael and other staff of General Hospital Idekpa, Benue State of Nigeria for their time and effort during the implementation of the PITC strategy.

\section{Funding}

This study received no specific grant from any funding agency in the public, commercial or not-for-profit sectors.

\section{Availability of data and materials}

Findings for the current study were based on the data shown in the manuscript. Strategies for data collection are noted in the method section of the manuscript. Please contact the corresponding author for additional information on data availability.

\section{Authors' contributions}

FAO conceptualised and designed the study, organised data collection, performed the analysis, interpreted the results, and drafted the manuscript. AM contributed to the design of the study, interpretation of results, and critically revised the manuscript. PO, KEA, Jl, TTZ and AP contributed to the interpretation of the results, and critically revised the manuscript. All authors read and approved the final manuscript.

\section{Authors' information}

Felix A. Ogbo MHM, MPH (Hons)

Andrew Page PhD

John Idoko MBBS, FMCP

Pascal Ogeleka MHM; MPH

Andrew Morgaji PhD

Terver Zua Tule MBBS

Kingsley Agho MPH, PhD

\section{Competing interest}

The authors declare that they have no competing interests.

\section{Consent for publication}

Not applicable

\section{Ethics approval and consent to participate}

Ethical approval for this assessment (and subsequent publication of findings) was obtained from the Benue State Ministry of Health Human Research Ethics Committee. All patients and caregivers (on behalf of children 13-18 years) were aware that as part of clinical care, their demographic and clinical data were registered in paper-based clinic registers, stored in the hospital medical registry. Data employed in this assessment were anonymous, and the ethics committees did not require that the investigators sought written informed consent from patients based on the WHO PITC guidelines. All patients or caregivers (for patient under 18 years) provided verbal informed consent to health care workers before they were allowed to participate.

\section{Publisher's Note}

Springer Nature remains neutral with regard to jurisdictional claims in published maps and institutional affiliations.

\section{Author details}

${ }^{1}$ Centre for Health Research, School of Medicine, Western Sydney University, Campbelltown Campus, Locked Bag 1797, Penrith, NSW 2571, Australia.

${ }^{2}$ Departement of Psychology, Faculty of Social Science, Benue State University, PMB 102119, Makurdi, Nigeria. ${ }^{3}$ Department of Public Health, School of Public Health, College of Science, Health and Engineering La Trobe University, Bundoora, VIC 3083 Australia. ${ }^{4}$ School of Science and Health, Western Sydney University, Campbelltown Campus, Locked Bag 1797, Penrith, NSW 2571, Australia. ${ }^{5}$ Department of Medicine, Faculty of Medical Sciences, University of Jos, P.M.B 2084, Jos, Plateau State, Nigeria. ${ }^{6}$ Prevention of Maternal-to-Child Transmission of HIV Unit, Benue State Ministry of Health, State Secretariat, High Level, PMB 102093, Makurdi, Benue State, Nigeria. ${ }^{7}$ General Hospital Idekpa, Ohimini Local Government Area, Benue State Hospitals Management Board, Makurdi, Benue State, Nigeria.

Received: 5 October 2016 Accepted: 4 March 2017

Published online: 09 March 2017

\section{References}

1. Joint United Nations Programme on HIV/AIDS. Global AIDS updates 2016 Geneva: UNAIDS; 2016 
2. GBD 2015 HIV Collaborators. Estimates of global, regional, and national incidence, prevalence, and mortality of HIV, 1980-2015: the Global Burden of Disease Study 2015. The Lancet HIV. 2016;3(8):E361-87.

3. Joint United Nations Programme on HIV/AIDS - UNAIDS. Global Report: UNAIDS report on Global AIDS Epidemic. Geneva: Joint United Nations Programme on HIV/AIDS; 2013.

4. Marks G, Crepaz N, Janssen RS. Estimating sexual transmission of HIV from persons aware and unaware that they are infected with the virus in the USA. Aids. 2006;20(10):1447-50.

5. Kahn JO, Walker BD. Acute human immunodeficiency virus type 1 infection. N Engl J Med. 1998;339(1):33-9.

6. Celum CL, Buchbinder SP, Donnell D, Douglas JM, Mayer K, Koblin B, et al. Early human immunodeficiency virus (HIV) infection in the HIV Network for Prevention Trials Vaccine Preparedness Cohort: risk behaviors, symptoms, and early plasma and genital tract virus load. J Infect Dis. 2001;183(1):23-35.

7. US Department of Health and Human Services - panel on Clinical Practices for Treatment of HIV Infection. Guidelines for the use of antiretroviral agents in HIV-1-infected adults and adolescents. Washington, DC: US Department of Health and Human Services; 2006.

8. Weintrob AC, Giner J, Menezes P, Patrick E, Benjamin DK, Lennox J, et al. Infrequent diagnosis of primary human immunodeficiency virus infection: missed opportunities in acute care settings. Arch Intern Med. 2003;163(17):2097-100.

9. World Health Organization / Joint United Nation Programme on HIV/AIDS. Guidance on provider-initiated HIV testing and counselling in health facilities. Geneva: World Health Organization; 2007.

10. Fleming PL, Ward JW, Janssen RS, De Cock KM, Valdiserri RO, Gayle HD, et al. Guidelines for national human immunodeficiency virus case surveillance, including monitoring for human immunodeficiency virus infection and acquired immunodeficiency syndrome. MMWR Recomm Rep. 1999;48:1-28.

11. UNAIDS, WHO. UNAIDS/WHO policy statement on HIV testing. Geneva: UNAIDS; 2004.

12. Wanyenze RK, Nawavvu C, Namale Alice S, Mayanja B, Bunnell R, Abang B, Amanyire G, Sewankambo NK, Kamya MR. Acceptability of routine HIV counselling and testing, and HIV seroprevalence in Ugandan hospitals. Bull World Health Organ. 2008;86(4):302-9.

13. Kankasa C, Carter RJ, Briggs N, Bulterys M, Chama E, Cooper Ellen R, Costa C, Spielman E, Katepa-Bwalya M, M'soka T. Routine offering of HIV testing to hospitalized pediatric patients at university teaching hospital, Lusaka, Zambia: acceptability and feasibility. J Acquir Immune Defic Syndr. 2009;51(2):202-8.

14. Leon N, Naidoo Pren, Mathews Catherine, Lewin Simon, Lombard C. The impact of provider-initiated (opt-out) HIV testing and counseling of patients with sexually transmitted infection in Cape Town, South Africa: a controlled trial. Implementation Science. 2010;5:8.

15. Ijadunola K, Abiona T, Balogun J, Aderounmu A. Provider-initiated (Opt-out) HIV testing and counselling in a group of university students in lle-lfe, Nigeria. J Acquir Immune Defic Syndr. 2011;16(5):387-96.

16. Federal Ministry of Health - Nigeria. National Guidelines for the prevention of mother-to-child transmission of HIV. Abuja: Federal Ministry of Health Nigeria; 2010.

17. Weiser SD, Heisler M, Leiter K, Percy-de Korte F, Tlou S, DeMonner S, et al. Routine HIV testing in Botswana: a population-based study on attitudes, practices, and human rights concerns. PLoS Med. 2006;3(7), e261.

18. Bassett IV, Giddy J, Nkera J, Wang B, Losina E, Lu Z, et al. Routine voluntary HIV testing in Durban, South Africa: the experience from an outpatient department. J Acquir Immune Defic Syndr. 1999;46(2):181.

19. Institute for Health Metrics and Evaluation. Financing Global Health 2015: Development assistance steady on the path to new Global Goals. Seattle: Institute for Health Metrics and Evaluation (IHME); 2016.

20. Futterman D, Gilden D, Stafford S, Laurie M, Wolfson S. ACTS: assess, consent, test, support: A rapid, reality-based system for HIV counseling and testing. N Y. 2004.

21. Odimayo M, Adediran S, Araoye M. Socio-demographic characteristics of adults screened for HIV/AIDS in a rural community in Benue state, Nigeria. African Journal of Clinical and Experimental Microbiology. 2010;11(1):29-136.

22. Anyebe EE, Hellandendu JM, Gyong JE. Socio-demographic profile of people living with HIV/AIDS (PLWHA) in Idoma land, Benue state, Northcentral Nigeria: Implications for HIV/AIDS control. International Journal of Sociology and Anthropology. 2013;5(5):153.
23. Kayigamba FR, Bakker MI, Lammers J, Mugisha V, Bagiruwigize E, Asiimwe A, et al. Provider-Initiated HIV Testing and Counselling in Rwanda: Acceptability among Clinic Attendees and Workers, Reasons for Testing and Predictors of Testing. PloS One. 2014;9(4):e95459. doi:10.1371/journal.pone. 0095459.

24. Topp SM, Chipukuma JM, Chiko MM, Wamulume CS, Bolton-Moore C, Reid SE. Opt-out provider-initiated HIV testing and counselling in primary care outpatient clinics in Zambia. Bull World Health Organ. 2011;89(5):328-35.

25. Creek TL, Ntumy R, Seipone Khumo Smith M, Mogodi M, Smit M, Legwaila K, Molokwane I, Tebele G, Mazhani L. Successful introduction of routine opt-out HIV testing in antenatal care in Botswana. J Acquir Immune Defic Syndr. 2007;45(1):102-7.

26. Kharsany AB, Karim QA, Abdool Karim SS. Uptake of provider-initiated HIV testing and counseling among women attending an urban sexually transmitted disease clinic in South Africa-missed opportunities for early diagnosis of HIV infection. AIDS Care. 2010;22(5):533-7.

27. Wanyenze R, Kamya M, Liechty CA, Ronald A, Guzman DJ, Wabwire-Mangen F, et al. HIV counseling and testing practices at an urban hospital in Kampala. Uganda AIDS and Behavior. 2006;10(4):361-7.

28. Shinsasa O, Simbayi L, Nelson Mandela/HSRC study of HIV/AIDS. South African national HIV prevalence, behavioural risks and mass media household survey 2002. South Africa: Human Sciences Research Council Cape Town; 2002. p. 2002

29. Meiberg AE, Bos AE, Onya HE, Schaalma HP. Fear of stigmatization as barrier to voluntary HIV counselling and testing in South Africa. East Afr J Public Health. 2008:5(2):49-54.

30. Sanjana P, Torpey K, Schwarzwalder A, Simumba C, Kasonde P, Nyirenda L, Kapanda $P$, et al. Task-shifting HIV counselling and testing services in Zambia: the role of lay counsellors. Human Resource Health. 2009;7:44.

31. World Health Organization. Treat train retrain. Task shifting: global recommendations and guidelines. Geneva: World Health Organization; 2007.

32. Ogbo FA, Provider-Initiated HIV. Testing and Counselling In Sub-Saharan Africa: The Role of Lay Health Workers. Austin Journal of HIV/AIDS Research. 2015;2(1):1014.

33. Lo B, Wolf $L$, Sengupta $S$. Ethical issues in early detection of HIV infection to reduce vertical transmission. JAIDS-HAGERSTOWN MD-. 2000;25:S136-43.

34. Rennie S, Behets F. Desperately seeking targets: the ethics of routine HIV testing in low-income countries. Bull World Health Organ. 2006;84(1):52-7.

35. Federal Ministry of Health - Nigeria. National guidelines for pediatric HIV and AIDS treatment and care. Abuja: Federal Ministry of Health - Nigeria; 2007.

36. Obermeyer CM, Bott S, Bayer R, Desclaux A, Baggaley R. HIV testing and care in Burkina Faso, Kenya, Malawi and Uganda: ethics on the ground. BMC Int Health Hum Rights. 2013;13:6.

37. Sharma M, Ying R, Tarr G, Barnabas R. Systematic review and meta-analysis of community and facility-based HIV testing to address linkage to care gaps in sub-Saharan Africa. Nature. 2015;528(7580):S77-85.

38. Bahwere P, Piwoz E, Joshua MC, Sadler K, Grobler-Tanner CH, Guerrero S, et al. Uptake of HIV testing and outcomes within a Community-based Therapeutic Care (CTC) programme to treat severe acute malnutrition in Malawi: a descriptive study. BMC Infect Dis. 2008;8:1.

39. Suthar AB, Ford N, Bachanas PJ, Wong VJ, Rajan JS, Saltzman AK, et al. Towards universal voluntary HIV testing and counselling: a systematic review and meta-analysis of community-based approaches. PLoS Med. 2013;10(8), e1001496.

40. Ikuomola V. NACA tests for HIV in Benue. The Nation Online. 2016. Available from: http://thenationonlineng.net/naca-tests-for-hiv-in-benue/.

41. Bila B, Egrot M. Gender asymmetry in healthcare-facility attendance of people living with HIV/AIDS in Burkina Faso. Soc Sci Med. 2009;69(6):854-61.

42. Galdas PM, Cheater F, Marshall P. Men and health help-seeking behaviour: literature review. J Adv Nurs. 2005:49(6):616-23.

43. Kaur M, Sodhi SK, Kaur P, Singh J, Kumar R. Gender differences in health care seeking behaviour of tuberculosis patients in Chandigarh. Indian J Tuberc. 2013:60:217-22.

44. Hjelm K, Atwine F. Health-care seeking behaviour among persons with diabetes in Uganda: an interview study. BMC Int Health Hum Rights. 2011;11:1. 